\title{
Mapping Wigner distribution functions into semiclassical distribution functions
}

\author{
G. W. Bund ${ }^{1, *}$ and M. C. Tijero ${ }^{2, \dagger}$ \\ ${ }^{1}$ Instituto de Física Teórica, Universidade Estadual Paulista, Rua Pamplona, 145, 01405-900 São Paulo, SP, Brazil \\ ${ }^{2}$ Pontifícia Universidade Católica de São Paulo, Rua Marquês de Paranaguá, 111, 01303-000 São Paulo, SP, Brazil
}

(Received 1 April 1999; published 19 April 2000)

\begin{abstract}
A mapping that relates the Wigner phase-space distribution function of a given stationary quantum mechanical wave function, a solution of the Schrödinger equation, to a specific solution of the Liouville equation, both subject to the same potential, is studied. By making this mapping, bound states are described by semiclassical distribution functions still depending on Planck's constant, whereas for elastic scattering of a particle by a potential they do not depend on it, the classical limit being reached in this case. Following this method, the mapped distributions of a particle bound in the Pöschl-Teller potential and also in a modified oscillator potential are obtained.
\end{abstract}

PACS number(s): $03.65 . S q$

\section{INTRODUCTION}

The phase-space formulation of quantum mechanics began with a paper of Wigner [1]. Because the phase-space formulation offers a framework in which quantum problems can be treated using the classical language as much as it is allowed, it has been applied to many areas of physics. Among these areas we mention statistical physics [2], quantum optics [3], collision theory [4,5], nuclear physics [6], and nonlinear physics $[7,8]$.

The phase-space distribution functions are the main tool of the phase-space formulation of quantum mechanics and among them the Wigner distribution function (WDF) has been used with success, for instance, in the description of atom-molecule collision processes $[9,10]$, in nuclear physics $[6,11]$, and also for studying the classical limit of quantum mechanics $[12,13]$. It is with this last topic that this work is concerned. In Sec. II we summarize Ref. [12], where it is shown how to perform the mapping that relates the WDF of a given stationary quantum mechanical wave function, a solution of the Schrödinger equation, to a solution of the Liouville equation, both subject to the same potential. Two examples have already been considered in Ref. [12]: the infinite square well and the potential step. In Sec. III of this work we apply this same mapping to a particle bound in the Pöschl-Teller potential [14] and in Sec. IV to a particle in the ground state of a modified oscillator potential. The potentials used in Sec. III and Sec. IV are smooth and more appropriate for classical or semiclassical approximations than those of Ref. [12].

\section{MAPPING THE WDF TO A SEMICLASSICAL DISTRIBUTION FUNCTION}

The classical limit of quantum mechanics may be accomplished in two steps. First the mapping of the WDF to a semiclassical phase-space distribution function (SDF) is obtained, and in a second step the limit $\hbar=0$ is taken since the

\footnotetext{
*Electronic address: bund@axp.ift.unesp.br

†Electronic address: maria@axp.ift.unesp.br
}

SDF may still depend on $\hbar$. We consider only the first of those two steps (Ref. [12]; the abbreviation SDF here corresponds to CDF in that reference).

The WDF $\rho(q, p, t)$ satisfies the equation [5]

$$
\frac{\partial \rho}{\partial t}+\frac{p}{m} \frac{\partial \rho}{\partial q}+\int K\left(q, p-p^{\prime}\right) \rho\left(q, p^{\prime}, t\right) d p^{\prime}=0,
$$

where $(q, p)$ is a point in phase space. The kernel $K$ is given by

$$
\begin{aligned}
K\left(q, p-p^{\prime}\right)= & \frac{i}{\hbar} \int \frac{d v}{2 \pi \hbar} e^{(i / \hbar)\left(p-p^{\prime}\right) v}\left[V\left(q-\frac{v}{2}\right)\right. \\
& \left.-V\left(q+\frac{v}{2}\right)\right],
\end{aligned}
$$

$V(q)$ being the potential. Equation (2) becomes in the classical limit [5]

$$
\begin{aligned}
K_{c}\left(q, p-p^{\prime}\right) & =-\frac{i}{\hbar} \int \frac{d v}{2 \pi \hbar} e^{(i / \hbar)\left(p-p^{\prime}\right) v} v \frac{\partial V}{\partial q} \\
& =-\frac{\partial V}{\partial q} \frac{\partial}{\partial p} \delta\left(p-p^{\prime}\right),
\end{aligned}
$$

and Eq. (1) goes over into the Liouville equation

$$
\frac{\partial \rho_{c}}{\partial t}+\frac{p}{m} \frac{\partial \rho_{c}}{\partial q}-\frac{\partial V}{\partial q} \frac{\partial \rho_{c}}{\partial p}=0 .
$$

We are looking for a particular solution $\rho_{c}$ of Eq. (4) which may be considered the correct semiclassical approximation of a given $\rho$, a solution of Eq. (1). In order to obtain it we write the integral equation

$$
\begin{aligned}
\rho(q, p, t)= & \rho_{c}(q, p, t)-\int_{-\infty}^{\infty} d t^{\prime} \int d q^{\prime} d p^{\prime} \\
& \times G_{c}\left(q, p ; q^{\prime}, p^{\prime}, t-t^{\prime}\right) \int d p^{\prime \prime}\left[K\left(q^{\prime}, p^{\prime}-p^{\prime \prime}\right)\right. \\
& \left.-K_{c}\left(q^{\prime}, p^{\prime}-p^{\prime \prime}\right)\right] \rho\left(q^{\prime}, p^{\prime \prime}, t^{\prime}\right)
\end{aligned}
$$


relating Eqs. (1) and (4), where $G_{c}\left(q, p ; q^{\prime}, p^{\prime}, t-t^{\prime}\right)$ is the retarded Green's function, which satisfies the inhomogeneous Liouville equation

$$
\frac{\partial G_{c}}{\partial t}+\frac{p}{m} \frac{\partial G_{c}}{\partial q}-\frac{\partial V}{\partial q} \frac{\partial G_{c}}{\partial p}=\delta\left(q-q^{\prime}\right) \delta\left(p-p^{\prime}\right) \delta\left(t-t^{\prime}\right)
$$

A WDF that satisfies Eq. (1) when inserted into Eq. (5) gives a function $\rho_{c}$ that satisfies Eq. (4) as can be verified by inserting Eq. (5) directly into Eq. (4). So Eq. (5) generates a SDF from a known WDF. This is the starting point for obtaining $\rho_{c}$. Using Eq. (1) and Eq. (3), Eq. (5) may be written

$$
\begin{aligned}
\rho_{c}(q, p, t)= & \rho(q, p, t)+\int_{-\infty}^{\infty} d t^{\prime} \int d q^{\prime} d p^{\prime} \\
& \times G_{c}\left(q, p ; q^{\prime}, p^{\prime}, t-t^{\prime}\right)\left(-\frac{\partial}{\partial t^{\prime}}-\frac{p^{\prime}}{m} \frac{\partial}{\partial q^{\prime}}\right. \\
& \left.+\frac{\partial V}{\partial q^{\prime}} \frac{\partial}{\partial p^{\prime}}\right) \rho\left(q^{\prime}, p^{\prime}, t^{\prime}\right) .
\end{aligned}
$$

We stress that Eq. (7) is equivalent to Eq. (5) if $\rho$ satisfies Eq. (1).

The WDF $\rho$ generated by a given wave function $\Psi(q, t)$, a solution of the Schrödinger equation corresponding to the potential $V(q)$, is given by [1]

$$
\rho(q, p, t)=\int \frac{d v}{2 \pi \hbar} e^{i p v / \hbar} \Psi\left(q-\frac{v}{2}, t\right) \Psi *\left(q+\frac{v}{2}, t\right) .
$$

In Eq. (8), when $\Psi$ is stationary, $\rho$ does not depend on the time. In the particular case in which $V(q)$ is quadratic in $q$, Eq. (7) reduces to $\rho=\rho_{c}$, as in this case Eq. (1) becomes identical to the Liouville equation.

The retarded Green's function satisfying Eq. (6) is

$$
\begin{aligned}
G_{c}\left(q, p ; q^{\prime}, p^{\prime}, \tau, \varepsilon\right)= & e^{-\varepsilon \tau} \delta\left(Q(q, p,-\tau)-q^{\prime}\right) \\
& \times \delta\left(P(q, p,-\tau)-p^{\prime}\right) \eta_{+}(\tau),
\end{aligned}
$$

where the convergence factor $\exp (-\varepsilon \tau)(\varepsilon \rightarrow 0)$, was introduced. Here $(Q(q, p, t), P(q, p, t))$ represents the classical trajectory in phase space of a particle subject to the potential $V(q)$, which at time $t=0$ occupies the phase-space point $(q, p)$, and $\eta_{+}$is the step function.

Using the classical equations of motion $P / \mathrm{m}$ $=\partial Q\left(q, p, t^{\prime}-t\right) / \partial t^{\prime}$ and $-\partial V / \partial Q=\partial P\left(q, p, t^{\prime}-t\right) / \partial t^{\prime}$ we get

$$
\begin{aligned}
& {\left[\left(\frac{\partial}{\partial t^{\prime}}+\frac{p^{\prime}}{m} \frac{\partial}{\partial q^{\prime}}-\frac{\partial V}{\partial q^{\prime}} \frac{\partial}{\partial p^{\prime}}\right)\right.} \\
& \left.\quad \times \rho\left(q^{\prime}, p^{\prime}, t^{\prime}\right)\right]_{q^{\prime}=Q\left(q, p, t^{\prime}-t\right), p^{\prime}=P\left(q, p, t^{\prime}-t\right)} \\
& =\frac{\partial}{\partial t^{\prime}} \rho\left(Q\left(q, p, t^{\prime}-t\right), P\left(q, p, t^{\prime}-t\right), t^{\prime}\right)
\end{aligned}
$$

and Eq. (7) becomes [12]

$$
\begin{aligned}
\rho_{c}(q, p, t)= & \rho(q, p, t)-\int_{-\infty}^{t} d t^{\prime} e^{-\varepsilon\left(t-t^{\prime}\right)} \\
& \times \frac{\partial}{\partial t^{\prime}} \rho\left(Q\left(q, p, t^{\prime}-t\right), P\left(q, p, t^{\prime}-t, t^{\prime}\right)\right) .
\end{aligned}
$$

When $\varepsilon$ may be set equal to zero from the beginning, we get from Eq. (11)

$$
\rho_{c}(q, p, t)=\lim _{t^{\prime} \rightarrow-\infty} \rho\left(Q\left(q, p, t^{\prime}\right), P\left(q, p, t^{\prime}\right), t^{\prime}\right) .
$$

Thus, if the limit in Eq. (12) does exist the value of $\rho_{c}$ at the phase-space point $(q, p)$ is the value of $\rho$ at the initial $(t=$ $-\infty)$ phase-space point of the classical trajectory that at time $t=0$ reaches the point $(q, p)$. Assuming that $\rho$ does not depend on the time, Eq. (11) gives, after integrating by parts, the mapping equation

$$
\rho_{c}(q, p)=\lim _{\varepsilon \rightarrow 0_{+}} \varepsilon \int_{-\infty}^{0} d \tau e^{\varepsilon \tau} \rho(Q(q, p, \tau), P(q, p, \tau)) .
$$

For systems with one degree of freedom at phase-space points where the classical energy $E(q, p)$ is negative, Eq. (12) cannot be applied and then Eq. (13) has to be used. Making the Fourier decomposition

$$
\rho(Q(q, p, \tau), P(q, p, \tau))=\sum_{n=-\infty}^{\infty} R_{n}(q, p) e^{i n \omega(q, p) \tau},
$$

where the period associated with the trajectory is $T(q, p)$ $=2 \pi / \omega(q, p)$, and inserting Eq. (14) into Eq. (13), we get $\rho_{c}(q, p)=R_{0}(p, q)$, which is equal to

$$
\rho_{c}(q, p)=\frac{1}{T(p, q)} \int_{0}^{T(q, p)} d \tau \rho(Q(q, p, \tau), P(q, p, \tau)),
$$

so the only nonvanishing term in the $\varepsilon=0_{+}$limit corresponds to $n=0$ in Eq. (14).

For points $(q, p)$ on the same closed classical path, Eq. (15) gives the same value of $\rho_{c}$, because this expression is invariant under time translations of the integrand, and the trajectories $(Q(q, p, t), P(q, p, t))$ associated with these points are related to each other by making time translations. This invariance shows that $\rho_{c}$ depends only on the energy 
$E(q, p)$ associated with the trajectory and it can be extended to open classical paths by making use of Eq. (13).

When the WDF corresponds to a bound state in a potential that vanishes at infinity, Eq. (12) may be applied directly for points with positive energy $E(q, p)$, giving the result $\rho_{c}(q, p)=0$. It can be shown also that the phase-space averaged classical energy for the SDF coincides with the quantum energy of the bound state. The mapped SDF in this case may be viewed as a stationary distribution function of classical particles trapped by the potential.

In order to see how this theory works two examples were considered.

\section{A. The infinite square well}

The ground state of the infinite square well

$$
V(q)=\left\{\begin{array}{cc}
0, & |q| \leqslant d \\
\infty, & |q|>d
\end{array}\right.
$$

is given by

$$
\Psi(q)=\frac{1}{\sqrt{d}} \cos \frac{\pi q}{2 d},|q| \leqslant d
$$

The WDF corresponding to this bound state is [4]

$$
\begin{aligned}
\rho(Q, P)= & \frac{1}{2 \pi \hbar d}\left\{\frac { \hbar } { 2 P } \left[\sin \left(\frac{2 P}{\hbar}(d-Q)+\frac{\pi}{d} Q\right)\right.\right. \\
& \left.+\sin \left(\frac{2 P}{\hbar}(d-Q)-\frac{\pi}{d} Q\right)\right] \\
& +\left(\frac{2 P}{\hbar}+\frac{\pi}{d}\right)^{-1} \sin \left[\left(\frac{2 P}{\hbar}+\frac{\pi}{d}\right)(d-Q)\right] \\
& \left.+\left(\frac{2 P}{\hbar}-\frac{\pi}{d}\right)^{-1} \sin \left[\left(\frac{2 P}{\hbar}-\frac{\pi}{d}\right)(d-Q)\right]\right\}
\end{aligned}
$$

for $0<Q<d$ and

$$
\rho(Q, P)=\rho(-Q, P)
$$

for $-d<Q<0$.

Making a change of variables in Eq. (15), we obtain for the SDF

$$
\rho_{c}(q, p)=\frac{1}{2 d} \int_{-d}^{d} \rho\left(q^{\prime}, p\right) d q^{\prime}=\frac{\pi}{4 \hbar} \frac{\cos ^{2}(p d / \hbar)}{\left[(\pi / 2)^{2}-(d p / \hbar)^{2}\right]^{2}} .
$$

We see that as the SDF $\rho_{c}$ depends only on the classical energy, which in this case is $p^{2} / 2 m$, it cannot depend on the coordinate $q$. In the limit $\hbar \rightarrow 0$ Eq. (20) gives $\rho_{c}(q, p)$ $\rightarrow(1 / 2 d)^{-1} \delta(p)$, which describes a uniform distribution of particles at rest inside a box.

\section{B. The potential step}

The wave function for the potential step

$$
V(q)=\left\{\begin{array}{cc}
0, & q<0 \\
V_{0}>0, & q \geqslant 0
\end{array}\right.
$$

for incident energies $E=k^{2} / 2 m<V_{0}$ is given by

$$
\psi(q)= \begin{cases}2 A \cos (k q / \hbar-\alpha / 2), & q<0 \\ 2 A \cos (\alpha / 2) e^{-\kappa q / \hbar}, & q>0\end{cases}
$$

where $\kappa=\left(2 m V_{0}-k^{2}\right)^{1 / 2}$ and $e^{i \alpha}=(i k+\kappa) /(i k-\kappa)$ gives the phase $\alpha$. The WDF is given by [4]

$$
\begin{aligned}
\rho(Q, P)= & 4 \frac{|A|^{2}}{\pi}\left[C^{(-)}(P, k) \cos \left(2(P-k) \frac{Q}{\hbar}\right)\right. \\
& +C^{(+)}(P, k) \cos \left(2(P+k) \frac{Q}{\hbar}\right) \\
& +S^{(-)}(P, k) \sin \left(2(P-k) \frac{Q}{\hbar}\right) \\
& \left.+S^{(+)}(P, k) \sin \left(2(P+k) \frac{Q}{\hbar}\right)\right],
\end{aligned}
$$

for $Q<0$, where

$$
\begin{aligned}
C^{\mp}(P, k)= & \kappa k\left[(\mp 2 P+k)^{2}+\kappa^{2}\right]^{-1}( \pm 2 P)^{-1}, \\
S^{\mp}(P, k)= & k\left( \pm 2 P k-k^{2}+\kappa^{2}\right) /\left[(\mp 2 P+k)^{2}+\kappa^{2}\right] \\
& \times 4 P(k \mp P) .
\end{aligned}
$$

For $Q>0$ the WDF is

$$
\begin{aligned}
\rho(Q, P)= & 4 \frac{|A|^{2}}{\pi} e^{-2 \kappa Q}\left[C_{0}(P, k) \cos \left(\frac{2 P Q}{\hbar}\right)\right. \\
& \left.+S_{0}(P, k) \sin \left(\frac{2 P Q}{\hbar}\right)\right],
\end{aligned}
$$

where

$$
\begin{gathered}
C_{0}(P, k)=4 \kappa k^{2}\left[(2 P+k)^{2}+\kappa^{2}\right]^{-1}\left[(2 P-k)^{2}+\kappa^{2}\right]^{-1}, \\
S_{0}(P, k)=\frac{k^{2}\left(k^{2}+\kappa^{2}-4 P^{2}\right)}{\left[(2 P+k)^{2}+\kappa^{2}\right]\left[(2 P-k)^{2}+\kappa^{2}\right] P} .
\end{gathered}
$$

The SDF that corresponds to the WDF given in Eqs. (23) and (25) is [12]

$$
\rho_{c}(q, p)=\left\{\begin{array}{cc}
|A|^{2}[\delta(p-k)+\delta(p+k)], & q<0 \\
0, & q>0 .
\end{array}\right.
$$

Equation (27) describes the reflection of a classical particle subject to the potential given by Eq. (21) for energies below the height of the barrier. Equation (27) can be written 


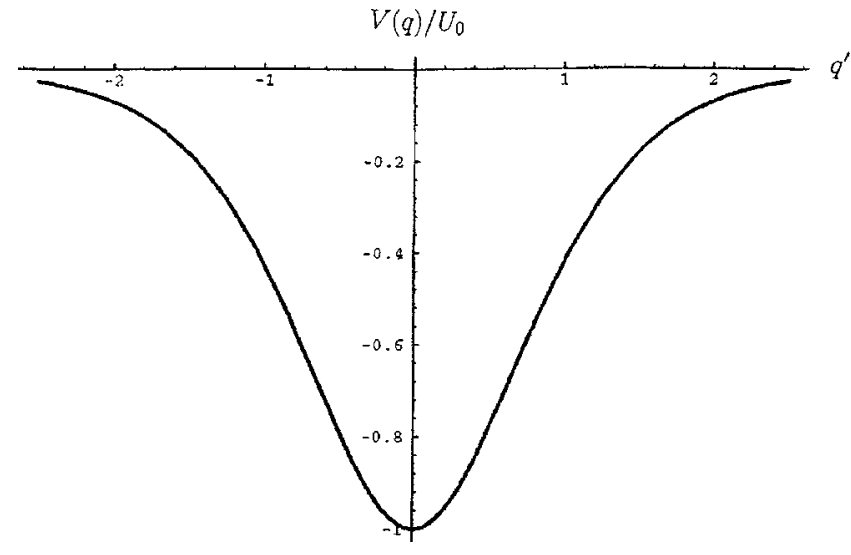

FIG. 1. The Pöschl-Teller potential as a function of the dimensionless variable $q^{\prime}=a q$.

$$
\rho_{c}(q, p)=\left\{\begin{array}{cc}
\frac{k|A|^{2}}{m} \delta\left(\frac{p^{2}}{2 m}-\frac{k^{2}}{2 m}\right), & q<0 \\
0, & q>0,
\end{array}\right.
$$

which explicitly shows the energy dependence of the SDF.

We remark that, in order to treat the potential step with a normalized wave function, the time dependent wave packet approach for scattering can be used [15]. The corresponding time dependent WDF and SDF are calculated afterwards. In the limit when the incident packet reduces to a single plane wave one expects the result of Eq. (27).

\section{THE PÖSCHL-TELLER POTENTIAL}

Here we present a third example, in which the mapping of the WDF corresponding to the ground state of the potential

$$
V(q)=-\frac{U_{0}}{\cosh ^{2}(a q)}, \quad U_{0}>0,
$$

into the SDF is performed. The parameters $U_{0}$ and $a$ are chosen such that the binding energy of the ground state becomes equal to $U_{0} / 2$. This choice fixes the value $U_{0}$ $=\hbar^{2} a^{2} / m$.

The normalized wave function, the solution of the Schrödinger equation corresponding to the potential given by Eq. (29), is

$$
\psi(q)=\left(\frac{a}{2}\right)^{1 / 2}[\cosh (a q)]^{-1}
$$

The WDF, which we obtained following Eq. (8), is

$$
\rho(q, p)=\frac{\sin \left(2 p q \hbar^{-1}\right)}{\hbar \sinh (2 a q) \sinh \left[\pi p(\hbar a)^{-1}\right]},
$$

which satisfies the condition $\int \rho(q, p) d q d p=1$.

Making $q=\pi q^{\prime} /(2 a)$ and $p=\hbar a p^{\prime}$ the expression (31) becomes symmetric in the dimensionless variables $q^{\prime}$ and $p^{\prime}$ (see Fig. 2). In Fig. 2 we draw the curves of constant density $\rho$ in the first quadrant; for the other quadrants the symmetry

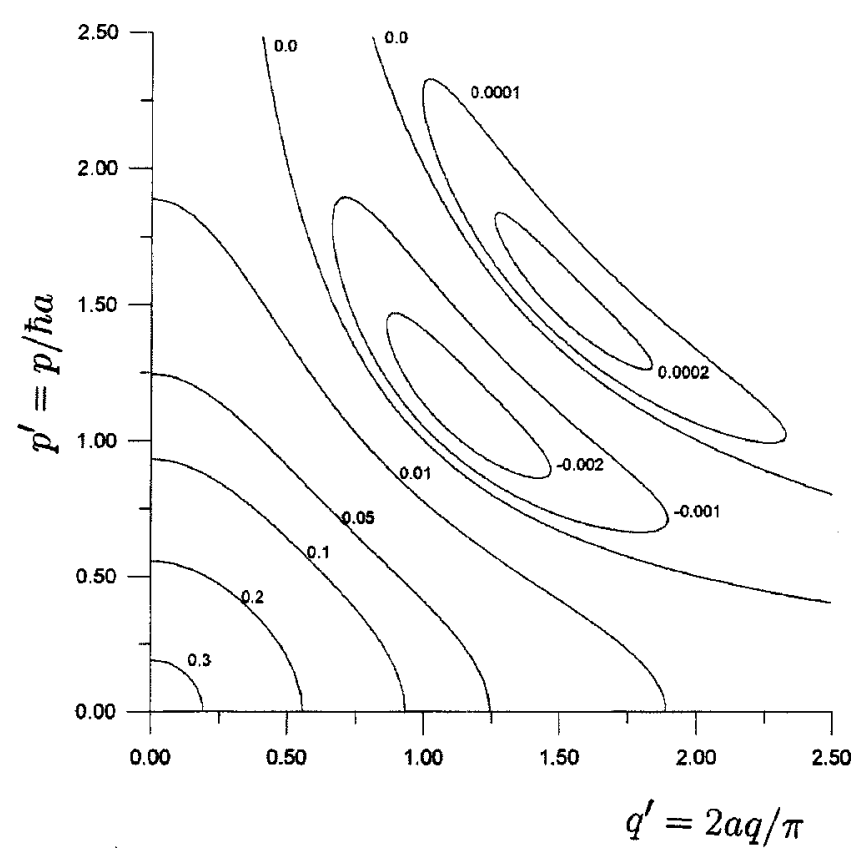

FIG. 2. Curves of constant density $\rho(q, p)$ (in units of $\hbar^{-1}$ ), the WDF corresponding to the ground state for the potential in Eq. (29). The variables $q^{\prime}$ and $p^{\prime}$ are dimensionless, $q$ and $p$ being the coordinate and momentum, respectively.

$q \rightarrow-q, p \rightarrow-p$ should be applied. We observe that regions in which $\rho$ is positive alternate with regions where it is negative. The points where $\rho(q, p)$ vanishes are given by the parabolas $2 p q=n \pi \hbar, n= \pm 1, \pm 2, \ldots$. The magnitude of $\rho$ in the region of negative values is small and occurs on points of phase space in which a classical particle would have positive energy, thus not being trapped by the potential.

The classical trajectories $(Q(q, p, t), P(q, p, t))$, the solutions of Newton's equations, are obtained by performing the integral

$$
\int d t=\int \frac{d Q}{\dot{Q}}=\int d Q\left(\frac{2 E}{m}+\frac{2 U_{0}}{m \cosh ^{2}(a Q)}\right)^{-1 / 2} .
$$

The constants of integration are determined by imposing the condition $(Q, P)=(q, p)$ at $t=0$.

For $E<0$ the result is

$$
\sinh (a Q)=\frac{a p \cosh (a q) \sin (\omega t)}{\omega m}+\sinh (a q) \cos (\omega t),
$$

$$
P \cosh (a Q)=p \cosh (a q) \cos (\omega t)-\frac{\omega m}{a} \sinh (a q) \sin (\omega t) .
$$

The frequency $\omega$ is related to the energy $E(q, p)$ of the trajectory by $\omega=a(2|E| / m)^{1 / 2}$. Equations (33) and (34) may be written

$$
\sinh (a Q)=\left(\frac{U_{0}}{|E|}-1\right)^{1 / 2} \sin \Phi(q, p, t),
$$




$$
P \cosh (a Q)=\left[2 m\left(U_{0}-|E|\right)\right]^{1 / 2} \cos \Phi(q, p, t),
$$

where

$$
\Phi(q, p, t)=\omega t+\varphi(q, p)
$$

Here

$$
\varphi(q, p)=\arctan [\omega m \sinh (a q) / a p \cosh (a q)] .
$$

The $\operatorname{SDF} \rho_{c}$ is obtained by replacing $(q, p)$ by $(Q(q, p, t), P(q, p, t))$ in Eq. (31) for the WDF and integrating it in $t$ over one period. For this purpose, expressions (35) and (36) are solved explicitly in $Q$ and $P$, the results being

$$
Q=a^{-1} \ln \left[\left(1+F^{2}\right)^{1 / 2}+F\right], \quad P=(2 m E)^{1 / 2} G\left(1+F^{2}\right)^{-1 / 2},
$$

where

$$
F=\left(U_{0} /|E|-1\right)^{1 / 2} \sin \Phi
$$

and

$$
G=\left(U_{0} /|E|-1\right)^{1 / 2} \cos \Phi
$$

We mention here that $\varphi(q, p)$ fixes the origin of the time and $\rho_{c}$ should not depend on it.

The final expression for $\rho_{c}$ for $E(q, p)<0$ is

$$
2 \pi \hbar \rho_{c}(q, p)=\int_{0}^{2 \pi} d \theta \frac{\sin \left\{2(a \hbar)^{-1}(2 m|E|)^{1 / 2} g\left(1+f^{2}\right)^{-1 / 2} \ln \left[\left(1+f^{2}\right)^{1 / 2}+f\right]\right\}}{2 f\left(1+f^{2}\right)^{1 / 2} \sinh \left\{\pi(2 m|E|)^{1 / 2} g\left[a \hbar\left(1+f^{2}\right)^{1 / 2}\right]^{-1}\right\}}
$$

where $f=\left(U_{0} /|E|-1\right)^{1 / 2} \sin \theta$ and $g=\left(U_{0} /|E|-1\right)^{1 / 2} \cos \theta$. Equation (38) shows that $\rho_{c}$ depends on $p$ and $q$ only through $E(q, p)$.

For positive energies $E(q, p)$ a similar calculation gives, as expected, $\rho_{c}(q, p)=0$, showing that the SDF corresponds indeed to a distribution of particles trapped inside the potential.

In Fig. 3 we plot the curves along which $\rho_{c}$ is constant (which coincide with the classical trajectories). The trajectory marked $\rho_{c}=0$ in Fig. 3 corresponds to $E(q, p)=0$. All

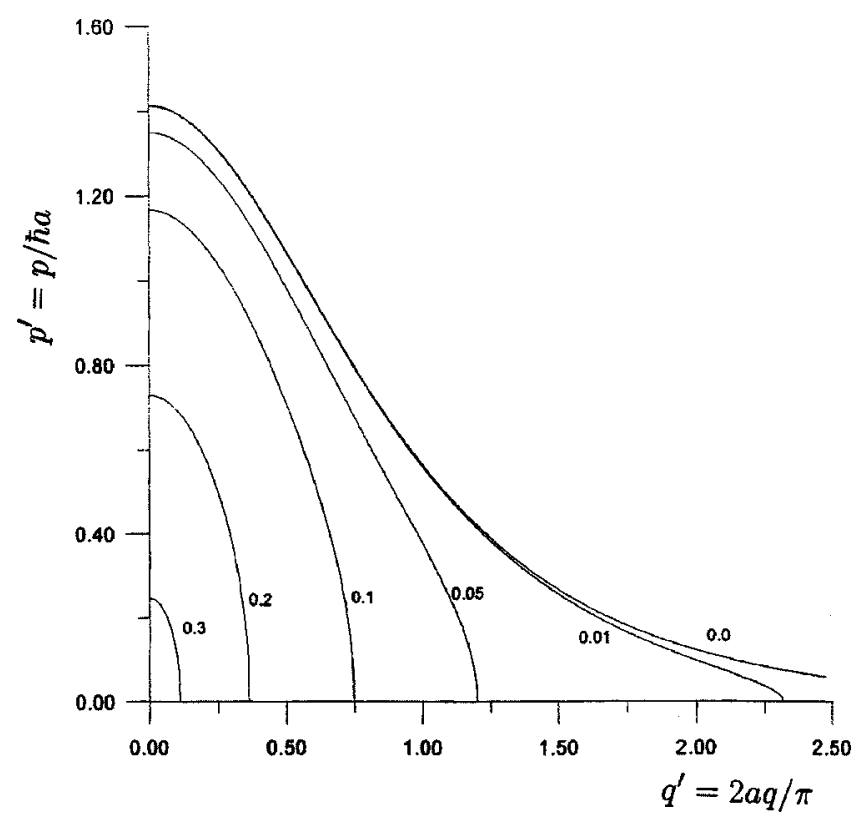

FIG. 3. Same as Fig. 2 for $\rho_{c}(q, p)$. In this case the curves are also the classical trajectories. The curve for which $\rho_{c}(q, p)=0$ corresponds to the energy $E(q, p)=0$. For points on the right of this curve $E(q, p)>0$ and $\rho_{c}=0$. trajectories for which $E(q, p)>0$ are situated at the right of this trajectory in this figure. As in the previous examples, we find also that only non-negative values for the density survive. Fig. 4 is a superposition of Fig. 2 and Fig. 3. In Fig. 4, when comparing the quantal curve for a given $\rho$ with the corresponding classical curve (same value of the density), one notices that the classical curve tends to move toward the left, away from the region corresponding to negative values of $\rho$. Thus, the region in which the WDF assumes negative values is located in the domain where $\rho_{c}$ vanishes.

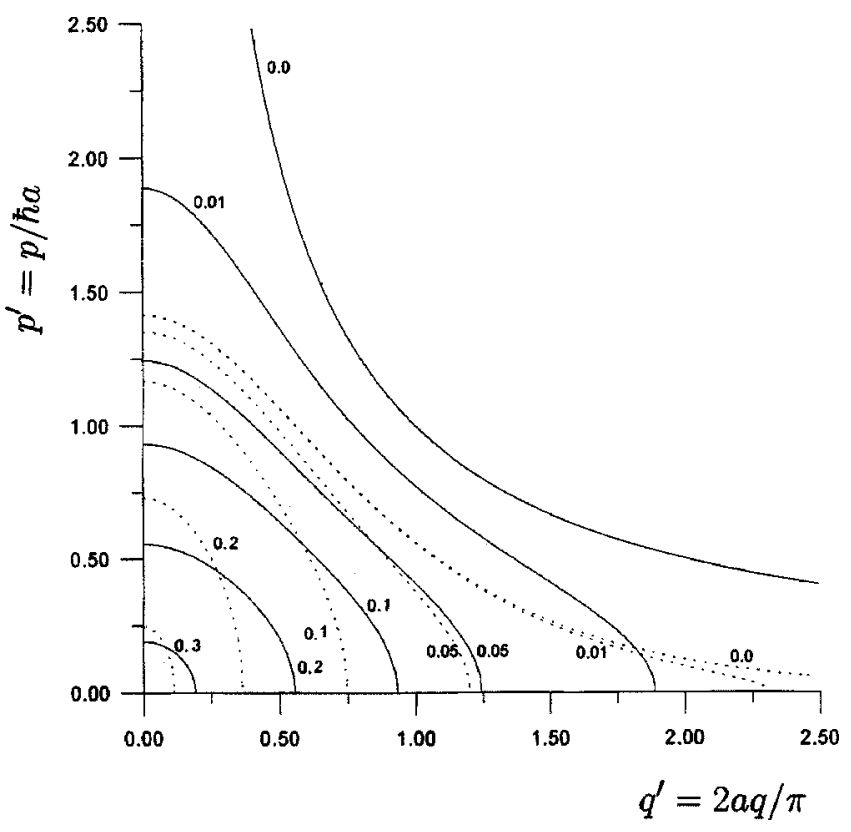

FIG. 4. Figures 2 and 3 are superposed. Dashed lines correspond to $\rho_{c}$ and full lines to $\rho$. Negative density curves are omitted. 


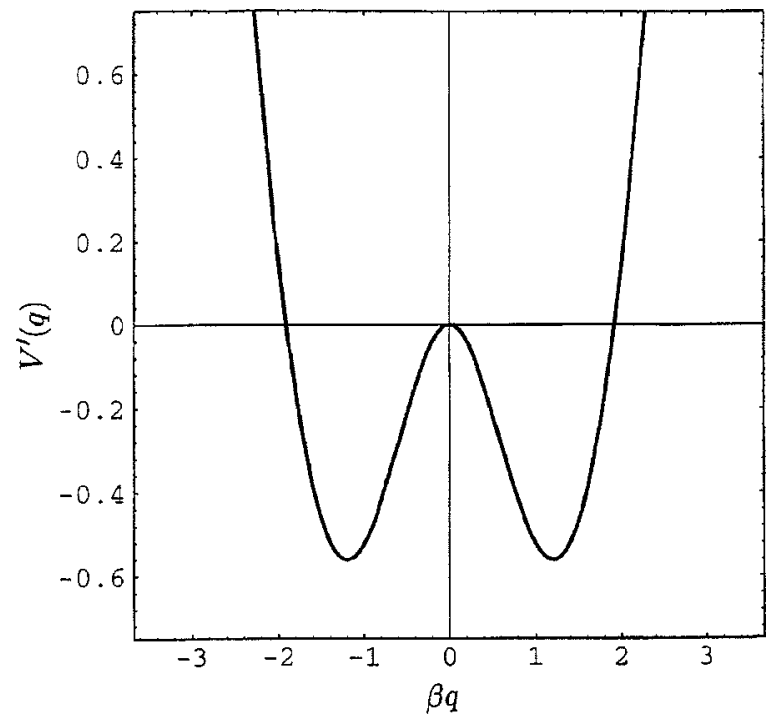

FIG. 5. The modified harmonic oscillator potential $V^{\prime}(q)$ $=2 m(\hbar \beta)^{-2} V(q), \beta q$ and $V^{\prime}(q)$ being dimensionless quantities.

\section{THE MODIFIED HARMONIC OSCILLATOR POTENTIAL}

Now we present results corresponding to the modified harmonic oscillator $(\mathrm{MHO})$ potential

$$
V(q)=\frac{\hbar^{2}}{2 m}\left[\alpha^{2} q^{2}-2 \alpha \beta q \tanh (\beta q)\right] .
$$

The wave function that corresponds to the ground state of this potential is

$$
\psi=N e^{\left(-\alpha q^{2} / 2\right)} \cosh (\beta q),
$$

with normalization factor $N^{2}=2(\alpha / \pi)^{1 / 2}\left[\exp \left(\beta^{2} / \alpha\right)+1\right]^{-1}$ and energy eigenvalue

$$
\epsilon(\alpha, \beta)=\frac{\hbar^{2}}{2 m}\left(\alpha-\beta^{2}\right) .
$$

The corresponding WDF may be calculated in closed form,

$$
\rho(q, p)=M e^{\left(-\alpha q^{2}-p^{2} / \alpha \hbar^{2}\right)}\left[e^{-\beta^{2} / \alpha} \cosh (2 \beta q)+\cos \left(\frac{2 \beta p}{\alpha \hbar}\right)\right],
$$

where $M=\left\{\pi \hbar\left[1+\exp \left(-\beta^{2} / \alpha\right)\right]\right\}^{-1}$ is the normalization factor. We shall consider $\alpha=\beta^{2}$, in which case we get $\epsilon(0,0)=0$ and the potential, given by Fig. 5, has a hump in the middle of the oscillator.

Equation (15) corresponding to the SDF $\rho_{c}$ was evaluated numerically making use of the expression

$$
\rho_{c}(q, p)=T^{-1} \oint \rho(Q, P(Q, E(q, p))) \frac{m d Q}{P(Q, E(q, p))},
$$

where the integration is performed over a closed path with energy $E(q, p)$. The period $T$ was simply calculated through

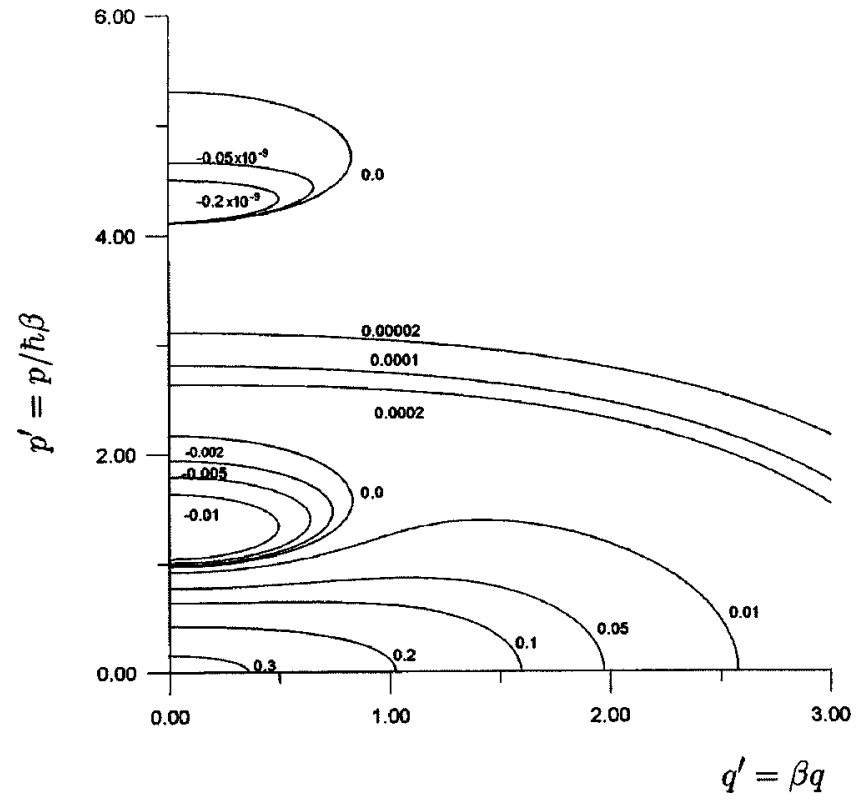

FIG. 6. Curves of constant density $\rho(q, p)$ (in units of $\hbar^{-1}$ ) for the WDF corresponding to the ground state of the modified harmonic oscillator potential. The variables $q^{\prime}$ and $p^{\prime}$ are dimensionless.

$$
T=\oint \frac{m d Q}{P(Q, E(q, p))},
$$

where $P(Q, E(q, p))$ is the momentum given as function of the coordinate $Q$,

$$
P(Q, E)=\{2 m[E-V(Q)]\}^{1 / 2} .
$$

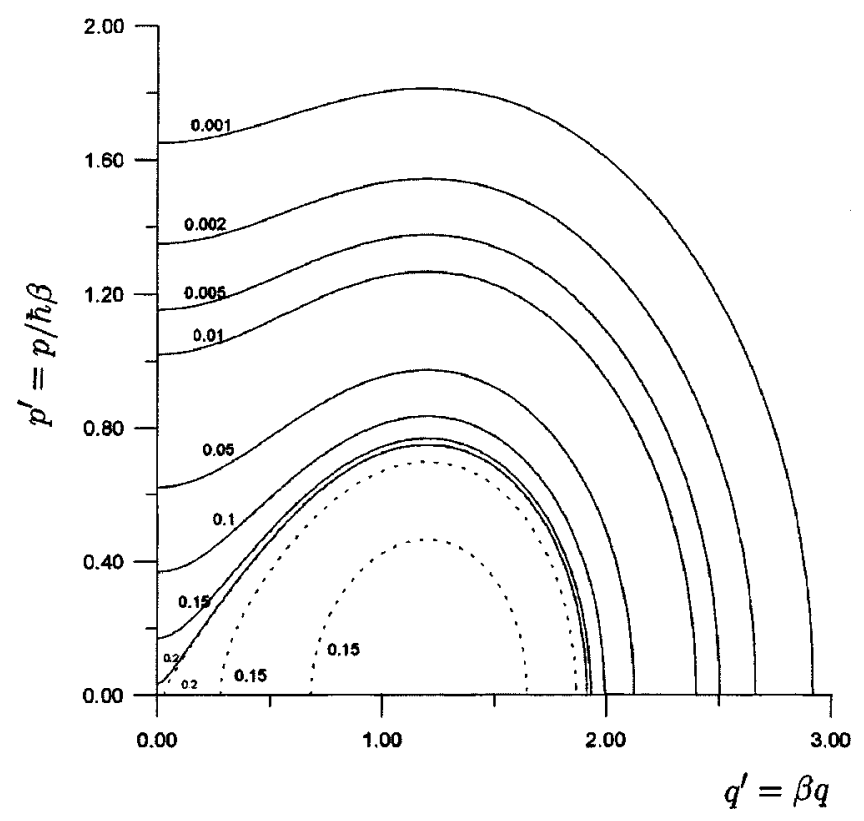

FIG. 7. Same as Fig. 6 for $\rho_{c}$. In this case the curves are also the classical trajectories. The dashed lines correspond to negative energies. 


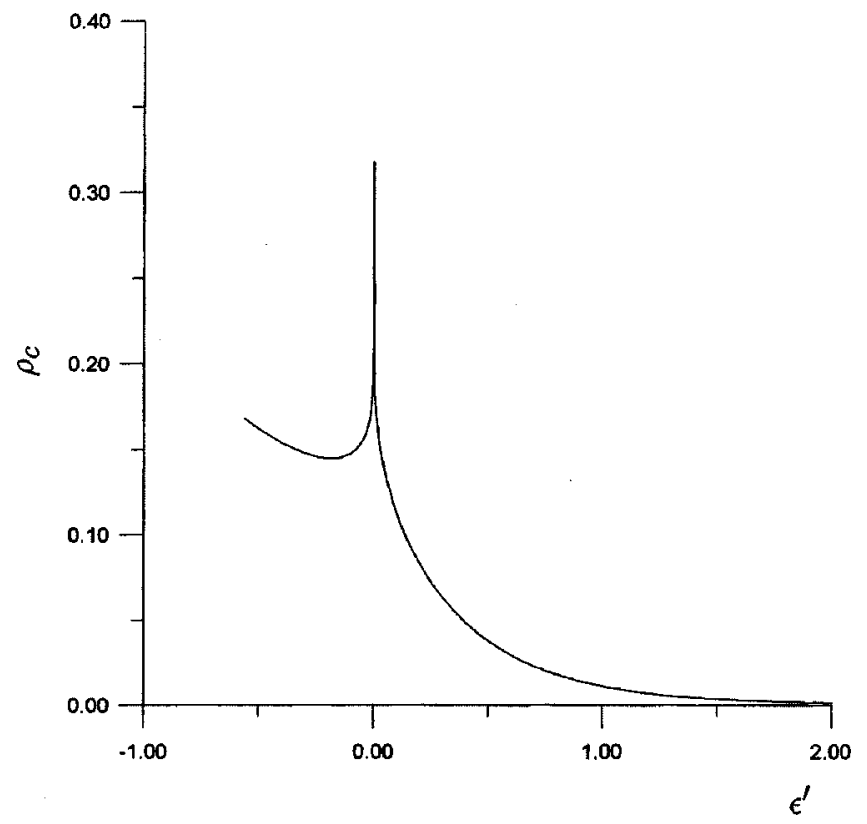

FIG. 8. The density $\rho_{c}$ (in units of $\hbar^{-1}$ ) of the SDF as function of the dimensionless classical energy $\epsilon^{\prime}(q, p)=E(q, p) 2 m(\hbar \beta)^{-2}$.

Equation (43) is obtained from Eq. (15) by replacing the time $\tau$ by the coordinate $Q$ as variable of integration. A second convenient change of variables $Q=Q(\theta)$ is made such that the singularities associated with the zeros of $P(Q, E)$ at the classical turning points are canceled by corresponding zeros in the numerator in Eqs. (43) and (44). Alternatively, we use the subtraction method for the elimination of these singularities in order to evaluate these integrals.

In Figs. 6 and 7 we plot, respectively, lines of constant $\rho$ and $\rho_{c}$ and in Fig. 8 we plot $\rho_{c}$ versus $E$, corresponding to the MHO potential for the choice $\alpha=\beta^{2}$. From Fig. 6 we see that the domain of phase space where $\rho$ is negative has its coordinates restricted to the region of the hump of the potential and it is periodic in the momentum variable. The value of $\rho$ decreases rapidly in magnitude as the momentum increases. As can be seen from Figs. 7 and 8, there may exist more than one trajectory for a given value of $\rho_{c}$. In Fig. 9 we superpose parts of Figs. 6 and 7, plotting curves with fixed density $\rho=\rho_{c}$. The SDF we obtain by applying Eq. (43) has similarities with the original WDF but again, as in the cases of Secs. II A, II B, and III, negative values are absent. One finds that for larger values of $q$ the two curves with $\rho=\rho_{c}=$ const tend to get closer, a result that is expected because of the dominance of the harmonic oscillator part of the potential.

We remark here that the point $(q, p)=(0,0)$, at the top of the hump, is a point of unstable equilibrium for classical

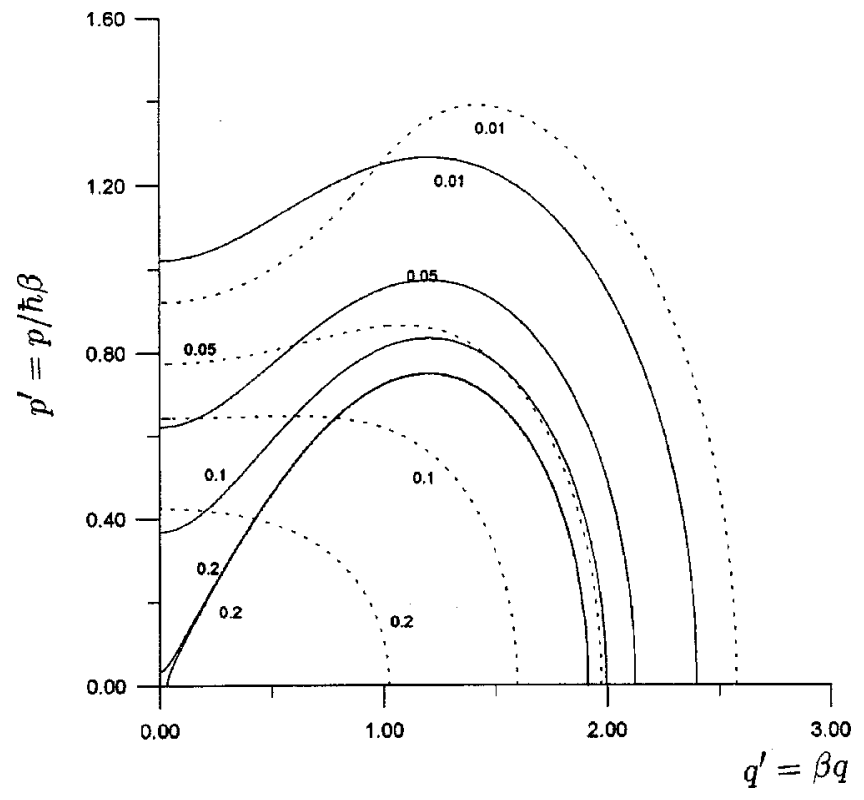

FIG. 9. Figures 6 and 7 are superposed. Dashed lines correspond to $\rho$ and full lines to $\rho_{c}$.

motion. Thus the period associated with the trajectory that passes through the point $(q, p)=(0,0)$ becomes infinite since the particle has to arrive at the point $q=0$ with zero velocity.

For this trajectory, which corresponds to $E=0$, the momentum $P(Q, 0)$ given by Eq. (45) is proportional to $Q$ in the neighborhood of the origin so that there is a pole at $Q=0$ in the integrand of Eq. (43) leading to a logarithmic singularity in the integral. This singularity is cancelled by the analogous singularity of $T$ given by Eq. (44). In fact, for phase-space points $(q, p)$ in the neighborhood of the trajectory $E(q, p)$ $=0$ the SDF is given by

$$
\hbar \rho_{c}=\frac{1}{\pi} \frac{A+\ln |E(q, p)|}{B+\ln |E(q, p)|},
$$

where $A$ and $B$ are slowly varying functions of $E(q, p)$. Thus one gets the value $\rho_{c}=1 / \pi$ for points on the trajectory $E(q, p)=0$. This value coincides with the maximum value of $\rho$, which occurs precisely at the point $(q, p)=(0,0)$. The functions $A$ and $B$ have to be evaluated numerically after subtraction of the pole term in the integrals in Eqs. (43) and (44). As can be seen in Fig. 8, Eq. (46) leads to a very sharp maximum of $\rho_{c}$ for the trajectory passing through the origin of phase space and for which $E(q, p)=0$ and $\hbar \rho_{c}=1 / \pi$. This path is compressed between the two curves for $\hbar \rho_{c}$ $=0.2$ in Figs. 7 and 9, the upper curve corresponding to $\varepsilon^{\prime}(q, p)=2 m(\hbar \beta)^{-2} E(q, p)=0.0010$ and the lower one to $\varepsilon^{\prime}(q, p)=-0.0010$.
[1] E. Wigner, Phys. Rev. 40, 749 (1932).

[2] J.E. Moyal, Proc. Cambridge Philos. Soc. 45, 99 (1949).

[3] R.J. Glauber, Phys. Rev. 131, 2766 (1963).

[4] H.W. Lee and M.O. Scully, Found. Phys. 13, 61 (1983).
[5] P. Carruthers and F. Zachariasen, Rev. Mod. Phys. 55, 245 (1983).

[6] G.P. Maddison and D.M. Brink, Nucl. Phys. A 378, 566 (1982). 
[7] M.V. Berry, Philos. Trans. R. Soc. London, Ser. A 287, 237 (1977).

[8] K. Takahashi, Suppl. Prog. Theor. Phys. 98, 109 (1989).

[9] H.W. Lee, Phys. Rep. 259, 147 (1995).

[10] G.W. Bund, S.S. Mizrahi, and M.C. Tijero, Phys. Rev. A 53, 1191 (1996).

[11] D. Galetti, A. Eiras, F.F. Souza Cruz, J.R. Marinelli, and M.M.
Watanabe de Moraes, Phys. Rev. C 48, 3131 (1993).

[12] G.W. Bund, J. Phys. A 28, 3709 (1995).

[13] S.D. Bosanac, Phys. Scr. 57, 171 (1998).

[14] L. D. Landau and E. M. Lifshitz, Quantum Mechanics (Pergamon, London, 1958), p. 69.

[15] E. Merzbacher, Quantum Mechanics (John Wiley, New York, 1998), p. 286. 